\title{
Finger Vein and Finger Dorsal Texture Recognition Joint Optimization based on Sparse Representation
}

\author{
Jun Li, Haiwei Liang and Wenming Yang ${ }^{\dagger}$ \\ Shenzhen Key Lab. of Information Sci\&Tech/Shenzhen Engineering Lab. \\ of IS\&DCP \\ Department of Electronic Engineering/Graduate School at Shenzhen, \\ Tsinghua University, China \\ †corresponding author, E-mail: yangelwm@163.com
}

\begin{abstract}
We introduce the sparse binary pattern (SBP), which is a feature extract method combined SRC with LBP.SBP is robust to illumination. In the paper, we put forward improvement in two directions: multi-value pattern and weighted pattern. Drawing lessons Finger Vein and Finger Dorsal Texture on LTP, we propose sparse ternary pattern (STP); considering different local block makes different contribution to finger vein and finger dorsal texture, we put forward the weighted sparse binary pattern (WSBP).Each improvement makes competitive results. At last, we consider the joint optimization of sparse multi-value pattern and Locally collaborative representation classification (LCRC),namely, we use sparse multi-value pattern in the feature extraction module and use or locally collaborative representation based classification (LCRC) in the classification module. The extensive experiments show that the joint optimization has more advantages than single optimization.
\end{abstract}

Keywords: sparse representation; sparse multi-value pattern; locally collaborative representation based classification; joint optimization

\section{Introduction}

This paper focuses on the Finger Vein and Finger Dorsal Texture recognition algorithm research based on sparse representation and collaborative expression, putting forward the corresponding improvement in feature extraction and classifier module of Finger Vein and Finger Dorsal Texture recognition system. In the feature extraction module, block processing the images of Finger Vein and Finger Dorsal Texture, taking larger weights for the obvious features of the texture image block, using sparse multi-value model for feature extraction.

In the classifier module, fusing and jointly optimizing the sparse binary mode and the expression method, to get more satisfactory results. 


\section{Finger Vein and Finger Dorsal Texture Database}

In order to evaluate the performance of the algorithm, it is necessary to test on the published finger vein database and finger vein database. We do algorithm testing based on the Tsinghua finger-vein and finger-dorsal image database.

At the time of acquisition, the finger is naturally straightened in the positioning device, and the image information of finger vein and finger dorsal texture are imaged at the same time.

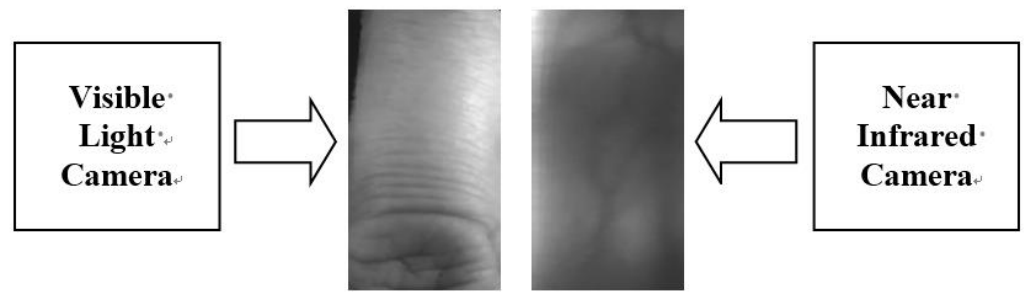

Fig.1. Image acquisition

As the blood vessels in the oxidation of hemoglobin and deoxygenated hemoglobin absorbs near-infrared light (cut-off frequency of $890 \mathrm{~nm}$, peak bandwidth of about $40 \mathrm{~nm}$ ), finger transmission image in venous distribution with information surrounding the brightness differences, were captured by cameras, form venous grayscale images. Ultimate finger dorsal texture image size is $100 \times 200$ at a resolution of 72 dpi. Finger veins digital visible direct imaging can be used with the same size and resolution. The database is divided into two subsets , Finger Dorsal Texture (FDT) and Finger Vein (FV),with each 610 samples were collected.

\section{Sparse multi-value pattern}

\subsection{Sparse binary pattern}

Local binary pattern (LBP) is a description method proposed for image texture, it is a classic feature extraction algorithm, put forward by Ojala in 1994. Sparse Binary patterns (SBP) is the combination of Sparse representation and local binary pattern, selecting a block which size is $\mathrm{N} \times \mathrm{N}$, in the pixel block, there are $(\mathrm{N}-2)^{2}$ center pixel values, getting the solution of the sparse representation by solving linear regression coefficients.

According to local consistency, select a $\mathrm{N} \times \mathrm{N}$ local block, which have $(\mathrm{N}-2)^{2}$ center pixel values $\mathrm{f}^{(\mathrm{k})}$, the vector form $\left.\mathrm{y}=\left[\mathrm{f}^{(1)}, \mathrm{f}^{(2)}, \ldots, \mathrm{f}^{(}(\mathrm{N}-2)^{2}\right)\right]^{\mathrm{T}}$, then

$$
f^{(\mathrm{k})}=\sum_{\mathrm{j}=1}^{8} \alpha_{\mathrm{j}} f_{\mathrm{j}}^{(\mathrm{k})}+\varepsilon_{\mathrm{k}}=\chi_{\mathrm{k}}^{\mathrm{T}} \alpha+\varepsilon_{\mathrm{k}}, \mathrm{k}=1,2, \ldots,(N-2)^{2}
$$


Among them, $f_{j}^{(k)}$ is the first $\mathrm{k}$ center of the first $\mathrm{j}$ neighboring pixels, each pixel values of the center can be expressed as $\chi_{\mathrm{k}}=\left[f_{1}^{(k)}, f_{2}^{(k)}, \ldots, f_{8}^{(k)}\right]$.

Write the linear equation of each center in the form of equations:

$$
\left[\begin{array}{c}
\mathrm{f}^{(1)} \\
\mathrm{f}^{(1)} \\
\cdots \\
\mathrm{f}^{(\mathrm{N}-2)^{2}}
\end{array}\right]=\left[\begin{array}{c}
\chi_{1}^{\mathrm{T}} \\
\chi_{2}^{\mathrm{T}} \\
\cdots \\
\chi_{(\mathrm{N}-2)^{2}}^{\mathrm{T}}
\end{array}\right] \alpha+\left[\begin{array}{c}
\varepsilon_{1} \\
\varepsilon_{2} \\
\cdots \\
\varepsilon_{(\mathrm{N}-2)^{2}}^{\mathrm{T}}
\end{array}\right]
$$

Its matrix forms are as follows

$$
\mathrm{y}=\mathrm{X} \alpha+\varepsilon
$$

So, it is converted to a multiple linear regression problem. In order to obtain better texture feature, and get a stable solution, this article uses the sparse linear regression. To simplify the texture expression, binarization processing the sparse coefficient.

$$
\beta_{j}= \begin{cases}1, & \alpha_{j}>0 \\ 0, & \text { otherwise }\end{cases}
$$

This is sparse binary pattern.

Through binarization processing, the SBP obtained is a binary bit string. The following shows how to similarly measure. There are many types of similarity measurement, this article selects Hamming distance and Cosine distance as instructions.

For the two binary bit string of $\beta^{1}$ and $\beta^{2}$, cosine similarity is defined as

$$
S_{c}\left(\beta^{1}, \beta^{2}\right)=\frac{1}{\left|\beta^{1}\right|\left|\beta^{2}\right|} \sum_{i=1}^{L} \beta_{i}^{1} \beta_{i}^{2}
$$

Hamming distance metric is defined as

$$
D_{\mathrm{H}}\left(\beta^{1}, \beta^{2}\right)=\sum_{\mathrm{i}=1}^{\mathrm{L}}\left|\beta_{\mathrm{i}}^{1}-\beta_{\mathrm{i}}^{2}\right|
$$

For the biggest hamming distance of the bit string is the length of the string, it can be defined hamming similarity

$$
\mathrm{S}_{\mathrm{H}}\left(\beta^{1}, \beta^{2}\right)=\left(1-\frac{\mathrm{D}_{\mathrm{H}}\left(\beta^{1}, \beta^{2}\right)}{\mathrm{L}}\right)
$$

\subsection{Sparse Ternary Patterns}

Inspired by the improvement of LBP, the sparse binary pattern also explore sparse multi-value pattern. Sparse binary pattern adopted the hard decisions, which have not strong robustness to noise. Then, explore the sparse ternary patterns (STB) to improve such defects.

Indicating function of defined the sparse ternary pattern 


$$
\beta_{j}= \begin{cases}0 & \alpha_{j} \leq 0 \\ 1 & 0<\alpha_{j} \leq t h \\ 2 & \alpha_{j}>t h\end{cases}
$$

Among them, $\mathrm{t}=[0, \mathrm{th}]$ is the defined threshold.

\subsection{Sparse binary Patterns}

In sparse binary pattern, each part of the image of finger vein pattern has different impact on the recognition rate. Texture feature of the areas other than the joint is not obvious, which has little contribution to the recognition rate. To make identification system make full use of texture feature, highlighting the large image block that has big contribution to the recognition rate, reducing local block which has not obvious texture feature, specifically putting forward the weighted sparse binary pattern(WSBP). WSBP divides the image into several equal rectangle shape, giving different weights in recognition of each image block. A method for determining weights is: take each local block image to recognize in turn, giving higher weights to high recognition rate.

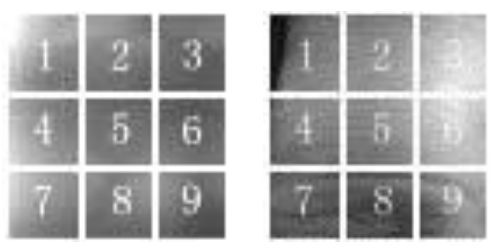

Fig.2.Local block diagram

\subsection{Experiments}

The experiment was conducted In FV and FDT Database of Tsinghua[9]. From the above analysis, it can be seen that the results of the Sparse Ternary Patterns and the weighted pattern are superior to the ordinary sparse binary patterns, if combining the ternary patterns and weighted patterns, can the obtained weighted sparse ternary patterns. Therefore, on the basis of former experiment, we have made further test and got the following results.

Table 1. Recognition rate of weighted sparse ternary patterns

\begin{tabular}{lllll}
\hline & \multicolumn{2}{l}{ OMP Optimization } & \multicolumn{2}{l}{ Lasso Optimization } \\
\cline { 2 - 5 } Database & FDT & FV & FDT & FV \\
& & & & \\
$\begin{array}{c}\text { Recognition } \\
\text { Rate }\end{array}$ & $\geq 0.9478$ & $\geq 0.9663$ & $\geq 0.9535$ & $\geq 0.9761$ \\
\hline
\end{tabular}




\section{Conclusion}

This paper starts from putting forward improvement the sparse binary pattern. For binary pattern with hard decision and the shortcoming of susceptible to noise interference, it puts forward the sparse ternary pattern(STP); For the different contribution of Finger Vein and Finger Dorsal Texture image, highlighting the bigger contributed local block and abandon the little contributed local block, putting forward the weighted sparse binary patterns(WSBP). Both improvement has got good results . Finally, combining these two kinds of improvement, it puts forward the weighted sparse ternary pattern(WSTP), which obtained more competitive results.

\section{Acknowledgement}

This work was supported by the National Key Research and Development Program of China with No.2016YFB0101001 in part, the Special Foundation for the Development of Strategic Emerging Industries of Shenzhen under Grant No.JCYJ20150831192224146 and National Science Foundation of China under Grant No.61471216.

\section{References}

1. Yang H, Wang Y. A LBP-based face recognition method with hamming distance constraint. Proceedings of Image and Graphics, 2007. ICIG 2007. Fourth International Conference on. IEEE, 2007. 645-649.

2. Liao S, Chung A C. Face recognition by using elongated local binary patterns with average maximum distance gradient magnitude. Proceedings of Computer Vision-ACCV 2007.Springer, 2007: 672-679.

3. Nanni L, Lumini A, Brahnam S. Local binary patterns variants as texture descriptors for medical image analysis[J]. Artificial intelligence in medicine, 2010, 49(2): 117-125.

4. Wright J, Yang AY, Ganesh A, et al. Robust face recognition via sparse representation [J]. Pattern Analysis and Machine Intelligence, IEEE Transactions on, 2009, 31(2): 210-227.

5. Zhang D, Yang M, Feng X. Sparse representation or collaborative representation: Which helps face recognition?[C]//Computer Vision (ICCV), 2011 IEEE International Conference on. IEEE, 2011:471-478.

6. Tropp, J.A. Gilbert,A.C. Signal Recovery From Random Measurements Via Orthogonal Matching Pursuit. Information Theory, IEEE Transactions, 2007, 53(12):4655-4666. 
7. Huang K, Aviyente S. Sparse representation for signal classification[C]//Advances in neural information processing systems. 2006: 609-616.

8. Yang M, Zhang D, Feng X. Fisher discrimination dictionary learning for sparse representation [C]//Computer Vision (ICCV), 2011 IEEE International Conference on. IEEE, 2011: 543-550.

9. http://www.sz.tsinghua.edu.cn/labs/vipl/thu-fvfdt.html 